\title{
Selected Results of the Collaborative Research Center "Droplet Dynamics under Extreme Ambient Conditions" SFB/TRR 75
}

\author{
Cameron Tropea*1, Bernhard Weigand ${ }^{2}$, Kathrin Schulte ${ }^{2}$ \\ ${ }^{2}$ Institute of Aerospace Thermodynamics, University of Stuttgart, Germany \\ ${ }^{1}$ Institute of Fluid Mechanics and Aerodynamics, Techn. Universität Darmstadt, Germany \\ *Corresponding author: ctropea@sla.tu-darmstadt.de
}

\begin{abstract}
The Collaborative Research Center (CRC) SFB-TRR 75 was established in January 2010 to focus on the dynamics of basic drop processes, and in particular on processes involving extreme boundary conditions, for example, near thermodynamic critical conditions, very low temperatures, under strong electric fields or in situations involving extremely large gradients. The CRC is a joint initiative of the University of Stuttgart, the TU Darmstadt and the German Aerospace Center (DLR) in Lampoldshausen, operating with 17 projects structured into three main research areas and involving researchers from numerous faculties: Mathematics, Chemistry, Electrical Engineering, Aerospace Engineering, Mechanical Engineering, Informatics and Computer Sciences. Some of the topics pursued at the $\mathrm{CRC}$ include
\end{abstract}

- The behaviour of supercooled and potentially electrified droplets in clouds

- $\quad$ The impact of Supercooled Large Droplets (SLD) on aircraft icing

- The behaviour of strongly electrified drops on insulator surfaces, which can be found on high voltage power lines, affecting the partial discharge behaviour and performance and durability of the insulator.

- Trans-critical injection conditions of fuel with flash boiling in rocket combustion chambers

- Atomization and vaporization of droplets at high pressures and temperature, as occurring in future combustion systems

This article provides an overview of the projects being carried out at the SFB-TRR 75 and highlights scientific results from selected subprojects. The main purpose of the paper is to familiarize colleagues with this extensive and dedicated research effort in the area of drop dynamics and to motivate and initiate future collaboration with others in this field.

Keywords: drop dynamics, extreme boundary conditions, supercooled large droplets, spray combustion, rocket engine sprays, flash boiling.

\section{Introduction}

Drops and drop dynamics are ubiquitous, in nature, e.g. in clouds, fog, rain, or spindrift, and in many engineering systems e.g. in gasoline or Diesel engines, gas turbines, aero-engines, rocket engines, spray cooling processes, chemical reactors, or agricultural sprays. Despite the fact that interest and research into the behaviour of droplets has begun very early, e.g. (Plateau, 1873) (Rayleigh, 1878) many fundamental aspects of droplet behaviour and drop dynamics remain unclear, especially under extreme ambient conditions. Under such extreme ambient conditions, the prediction and simulation of drop behaviour is often unreliable, moreover, standard literature on the topic of drop dynamics, e.g. (Lefebvre, 1989) (Pruppacher \& Klett, 1978) (Frohn \& Roth, 2000) often treat drops under only moderate ambient conditions. Therefore, in the particular area of drops under extreme ambient conditions different topics were identified for further basic research, with the aim to improve our understanding and predictive capabilities of both naturally occurring and engineering systems involving droplets.

Following the insight that complex droplet dynamic processes are determined by the interaction of very fundamental processes, the first 4-year period of the SFB-TRR 75 was dedicated to the behaviour of single droplets. In the current period, which runs from 2014-2017, the physical understanding and the methodology gained in the first phase are applied to small clusters of droplets to better understand their interaction. In the final period of the CRC in the years 2018 - 2021, the research will focus on ensembles of drops, or sprays.

The SFB-TRR75 is structured into three main research areas comprising 17 subprojects, listed below together with the responsible researcher(s):

\section{Research Area A: Methods and Fundamentals}

- TP-A1 Interactive visualization of droplet dynamic processes (T. Ertl / F. Sadlo)

- TP-A2 Development of numerical methods for the simulation of compressible droplet dynamic processes under extreme conditions (C.-D. Munz) 
- TP-A3 Analysis and numerics of front and phase field models for droplet dynamics (C. Rohde / V. Schleper)

- TP-A4 Molecular dynamics simulations of droplet evaporation in the non-linear response regime (F. MüllerPlathe)

- TP-A5 Simulation of the mechanical deformation and movement of droplets under the influence of high electric fields (T. Weiland / E. Gjonaj)

- TP-A6 Development and application of new thermodynamic models for interfaces based on classical density functional theory (J. Groß)

- TP-A7 Modelling and simulation of droplet collisions and explosions of droplets at high pressure and far from equilibrium (D. Bothe)

\section{Research Area B: Free Droplets}

- TP-B1 Investigation of the behaviour of supercooled droplets concerning evaporation, condensation and freezing at different boundary conditions (B. Weigand / N. Roth)

- TP-B2 Experimental investigation of droplet evaporation under extreme conditions by temporally highly resolved laser diagnostic methods (G. Lamanna / A. Dreizler)

- TP-B3 Modelling and simulation of droplet evaporation in different gas environments under supercritical conditions (A. Sadiki / J. Janicka)

- TP-B4 Experimental investigation of transient injection phenomena in rocket combusters at vacuum with flash evaporation (M. Oschwald / C. Manfletti)

- TP-B5 Modelling and simulation of the flash evaporation of cryogenic liquids (A. Kronenburg)

\section{Research Area C: Droplets with Wall-Interactions}

- TP-C1 Numerical simulation of the transport processes during drop impingement onto heated walls with special consideration of the evaporating three-phase contact line (T. Gambaryan-Roisman / P. Stephan)

- TP-C2 High resolution measurements of heat transfer during drop impingement onto a heated wall with particularly consideration of evaporation at the three phase contact line (P. Stephan I T. Gambaryan-Roisman)

- TP-C3 Impact of supercooled droplets onto cold surfaces (S. Jakirlic / C. Tropea)

- TP-C4 Interaction of a single drop with a heated wall at high ambient pressures (I. Roisman / C. Tropea)

- TP-C5 Mechanical and electrical phenomena of droplets under the influence of high electric fields (V. Hinrichsen)

In Research Area A numerical and analytical methods are developed which are pre-requisites for conducting the work in the other research areas. Methods for visualization are developed in TP-A1. TP-A2 and TP-A3 are concerned with the development of numerical methods. In TP-A4 basic droplet dynamic processes are investigated by molecular thermodynamics. In TP-A5 simulations of the mechanical deformation and movement of droplets on polymer insulation surfaces with strong electric fields are investigated. TP-A6 investigates the nonequilibrium thermodynamics of interfaces. Here the classical density functional theory is applied to mixtures of polar substances. In TP-A7 the focus is set on droplet collision processes.

In Research Area B droplets free of solid boundaries are investigated. In TP-B1 droplet dynamics problems involving supercooled droplets in clouds and the formation of ice crystals in clouds are investigated. TP-B2 investigates experimentally the evaporation of droplets under extreme thermodynamic conditions. The modelling and simulation of droplet evaporation in a foreign-gas environment under transcritical conditions are investigated in TP-B3, whereas the project TP-B4 investigates flash evaporation for the transient injection of propellant in a rocket combustion chamber. Project TP-B5 focuses on the numerical simulation of flash evaporation processes in strong cooperation with TP-B4.

In Research Area C droplet-wall interactions are investigated. In TP-C1 and TP-C2 special focus is set on the three-phase contact line during droplet-wall interaction. In TP-C3 droplet-wall interactions are investigated for supercooled droplets on cold surfaces and in TP-C4 droplet-wall interaction is investigated for hot surfaces under high ambient pressures.

Results from this CRC have been reported previously in (Weigand \& Tropea 2014, 2015, 2016); the present article presents further results and progress in selected subprojects. 


\section{Electrohydrodyamic simulation of electrically controlled droplet generation (Cooperation TP-A5, TP-B1, TP-B2, TP-C3) Dreizler, Gjonaj, De Gersem, Lamanna, Ouedraogo, Preusch, Steinhausen, Weigand, Weiland}

In this study, the generation of drops from an electrically driven droplet generator is experimentally realized and numerically simulated. An electro hydrodynamic model for the simulation of droplet formation, detachment and motion in the droplet generator is introduced. The numerical approach is based on the coupled solution of the multiphase flow problem with the charge continuity equation. For the latter, a modified convection-conduction model is applied, taking into account conductive, capacitive as well as convective electrical currents in the fluid. This allows for a proper description of charge relaxation phenomena in the moving fluid. In particular, the charge received by the droplet after detachment is an important parameter influencing the droplet dynamics in the test chamber. Simulation results are shown for highly conductive acetone droplets and for low conductivity pentane droplets, respectively. The operation characteristic of the droplet generator is investigated by computing droplet sizes and detachment times with respect to the applied voltage. Further details of this work, in particular about the solved equations and numerical solution procedure, can be found in Ouedraogo et al., 2017. The presentation here will focus only on selected results.

Electric fields can be used for controlled droplet generation, allowing for a reliable injection of liquid samples into a test chamber, even under extreme atmospheric conditions (Weckenmann et al., 2011). The strong electric field applied to a suspended droplet at the tip of a capillary produces the necessary momentum kick, causing the droplet to detach with a given volume and at a well-defined time instant. However, the process of droplet formation, its detachment from the tip of the capillary and, later, the droplet motion in the chamber represent a highly nonlinear and coupled problem, which can be described accurately only by means of numerical simulation. Such a detailed characterization of the droplet dynamics is necessary, in particular, for the prediction of device behaviour for different applied voltages, liquid types, and chamber atmospheric conditions.

(a)

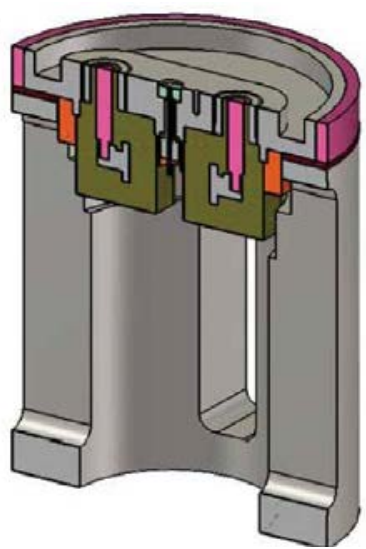

(b)

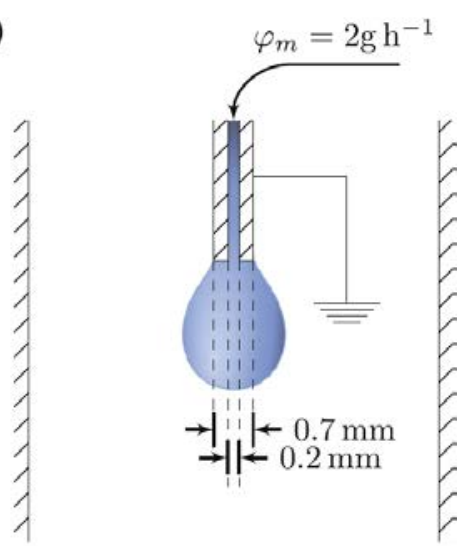

Figure 1: (a) Full model of the droplet generator including capillary, electrodes and test chamber. (b) Schematic view and main parameters of the simulation model

The complete generator setup is shown in Figure 1(a). Its main components are a metallic capillary tube and two high voltage electrodes. The capillary is kept at ground potential, whereas the electrodes are connected to a highvoltage source. In order to prevent electric breakdown, the latter are embedded in two large insulator blocks. Both, the capillary and the electrodes are installed in a high-pressure test chamber capable of sustaining a high pressure, high temperature gaseous environment. The geometry of the droplet generator is not quite axisymmetric. Nevertheless, the electric field is nearly axisymmetric near the capillary tip. This is why a 2D-simulation approach is used.

Observations show that droplet detachment time and charging behaviour, as well as the flow dynamics after detachment depend strongly on the electrical properties of the liquid phase. This is due to the modification of the local electric field pattern by the droplet. This is illustrated in Figure 2 , where the electric field and force distribution is shown for a highly conductive acetone and for a low conductivity pentane droplet. In the low conductivity case, electric fields penetrate much deeper into the liquid, featuring a clear field singularity at the tip of the capillary. Contrary to this, the electric field within conductive acetone droplets nearly vanishes. Furthermore, the different electric properties of the two liquids result in a substantially different distribution of the electric force density on the droplet surface. In order to emphasize the different droplet detachment and charging behaviour these two cases were analysed by separate numerical experiments. In the following, only the results of the n-pentane drop generation will be presented. 

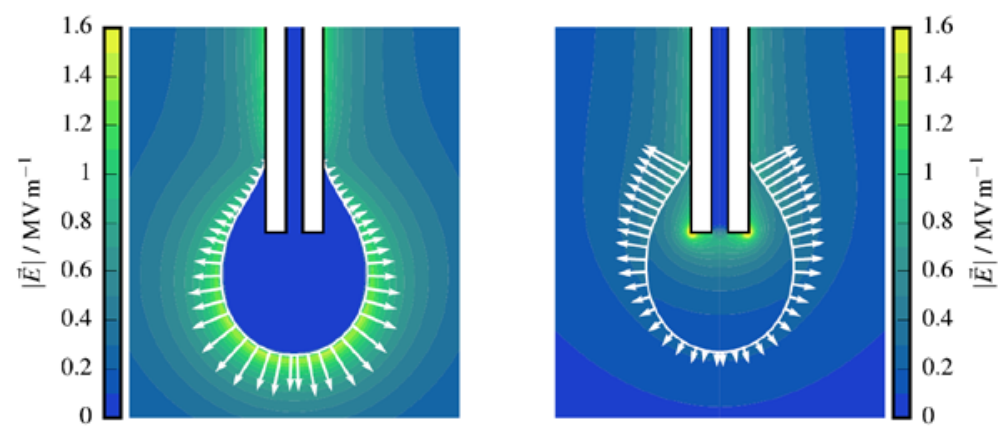

Figure 2: (a) Electric field and surface force distributions for pending (static) droplets assuming different electric properties of the liquid. (a): acetone (high conductivity) droplet. (b): n-pentane (low conductivity) droplet.

The assumed electric conductivity of $n$-pentane is $20 \mathrm{pS} / \mathrm{m}$, corresponding to a charge relaxation time of $\sim 1 \mathrm{~s}$. Since this relaxation time is much larger than a typical voltage pulse duration of $\sim 30 \mathrm{~ms}$, pentane droplets are always electrically charged. The results of the pentane droplet simulation are shown in Figure 3 . The test chamber is at temperature $27^{\circ} \mathrm{C}$ and pressure 1bar. A voltage pulse of $2.5 \mathrm{kV}$ and duration $26.5 \mathrm{~ms}$ is applied. The static contact angles were estimated to $\theta_{\mathrm{adv}}=10^{\circ}$ and $\theta_{\mathrm{rec}}=20^{\circ}$, respectively. As seen in Figure 3 , the agreement between simulation and experiment is nearly perfect. In particular, the liquid thread developing at detachment time (last frame in Figure 3) very closely resembles the experimentally recorded image. Furthermore, the simulation reveals a different behaviour in the n-pentane case compared to the acetone one. As seen in frames 3 to 4 of the figure (at times $40 \mathrm{~ms}$ to $75 \mathrm{~ms}$ ), the droplet is initially pushed up in the direction of the capillary when the external voltage is applied. When the voltage is switched off, the uncompensated weight of the droplet causes it to move down again and, eventually, detach. This is a completely different detachment mechanism than observed in the case of acetone droplets. It can be explained with the low electrical conductivity of $n$-pentane. In this case, electric fields penetrate deep into the liquid phase (see also Figure 2). Thus, electric charges are induced on the droplet surface as well as within the droplet and on the capillary tip. With the electric fields being concentrated close to the capillary tip in the upper half of the droplet, the effective electric polarization force points upwards, causing the droplet to ascend. This situation, however, may change again when the droplet bottom approaches the capillary tip. Thus, as long as the external voltage is not switched off, the droplet will perform oscillations around the capillary tip and may never detach.

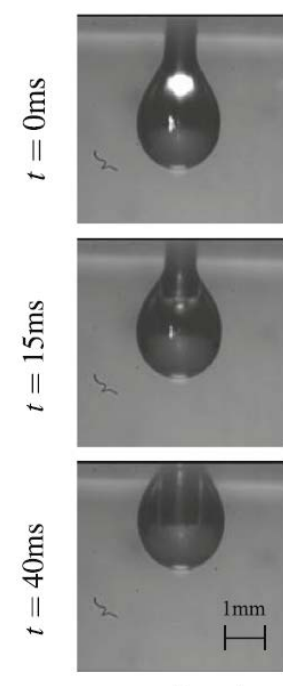

experiment

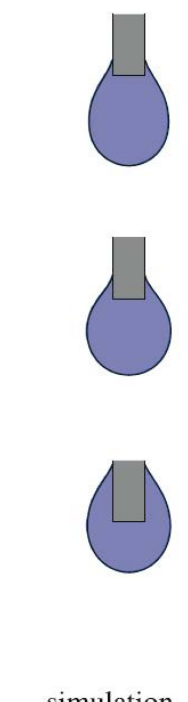

simulation
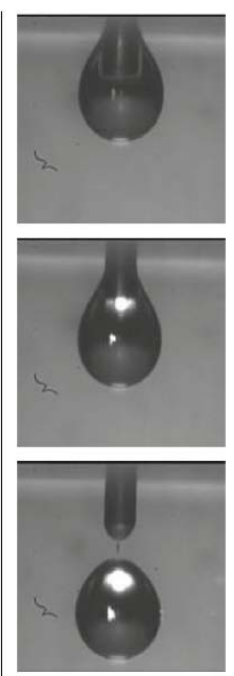

experiment
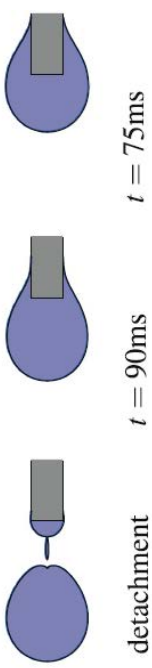

simulation

Figure 3: Pentane droplet dynamics: comparison between simulation and experiment for the droplet shape in the generator at different time instants during the detachment process.

In summary, the droplet charging effect is demonstrated for pentane droplets as well as for acetone droplets under long voltage pulses. This investigation shows that due to the very different relaxation times, the charging behaviour of the two liquids is very different. This behaviour is closely related to the drop dynamics in the generator. In particular, the detachment mechanisms for acetone and pentane droplets are completely different due to their electric properties. For low conductivity (pentane) droplets, the detachment is primarily due to the uncompensated droplet weight, which dominates droplet dynamics only after the generator voltage is switched off. 


\section{Effect of nano-textured heater surfaces on evaporation at a single meniscus (TP-C1, TP-C2) Fischer, Gambaryan-Roisman, Sahu, Sinha-Ray, Stephan, Yarin}

Nanofiber coatings have shown a unique potential for heat transfer enhancement during drop impact cooling, nucleate boiling and flow boiling. In order to gain insight into the mechanisms of heat transfer enhancement invoked by the nanofiber coating, the evaporation of liquid in the vicinity of an apparent contact line of a single meniscus, where the liquid-vapor interface meets the nanotextured substrate, has been investigated. Experiments have been performed for stationary, advancing and receding menisci. It has been found that the local heat flux near a stationary apparent contact line increases by approximately $60 \%$ in the presence of nanofiber coating. The receding meniscus leaves behind an extended region, in which the pores within the nano-textured mat are fully or partially filled with evaporating liquid, contributing significantly to heat transfer enhancement. These phenomena can be attributed to very strong capillary forces acting within the porous media and retaining the cooling liquid in contact with the heater surface. Further details of this work can be found in Fischer et al., 2017. The presentation here will focus only on selected results.

Increasing heat release from electronic devices and miniaturization of such devices pose serious challenges for heat removal from the heated surfaces. One of the promising and low-cost methods of surface modification is coating of a high-powered substrate with electrospun nanofibers, which has shown a significant increase in cooling rate during drop impact cooling, pool boiling and flow boiling in minichannels. The nanostructured surfaces are also capable of suppressing the Leidenfrost effect when coolant drops impact onto a high-temperature surface. The observed heat transfer enhancement during drop impact onto nanofiber mats has been attributed to two distinct phenomena: to hydrodynamic focusing,which prevents the liquid splashing after drop impact, and to imbibition of the liquid into the pores after the initial spreading phase. The imbibition of liquid into the porous structure leads to a two-order of magnitude increase of the substrate area available for evaporative cooling in comparison to the drop impact onto an uncoated substrate.

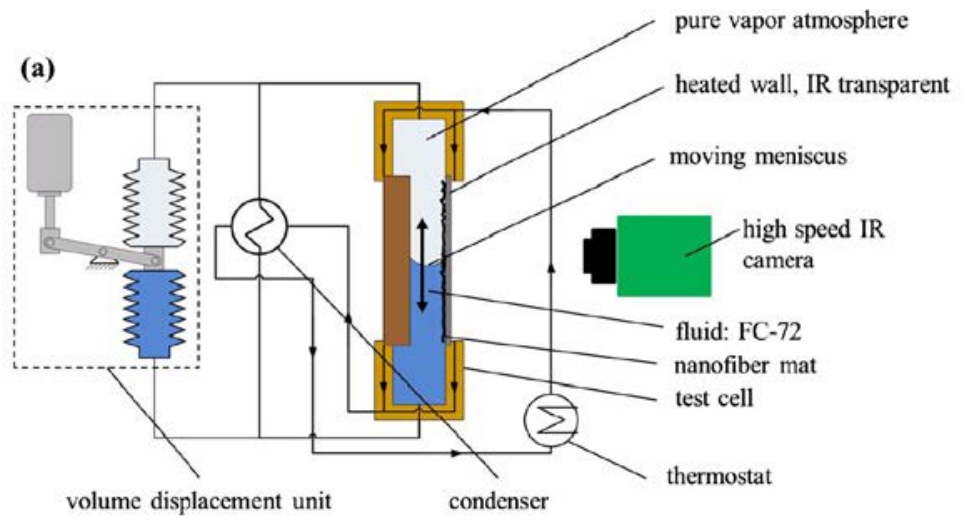

(b)

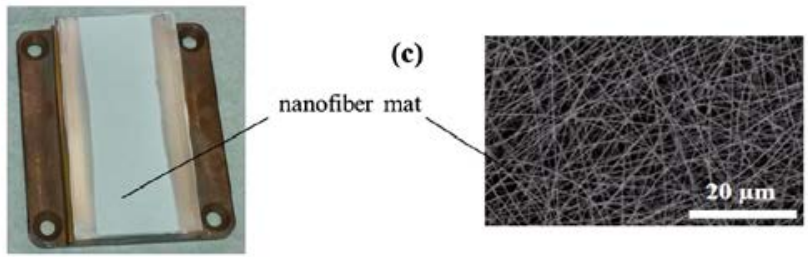

Figure 4: Experimental setup. (a) capillary slot apparatus. (b) target surface. (c) nanofiber mat.

A series of experiments has been performed using the experimental setup described in Fischer et al. (2015). The experimental setup is schematically shown in Figure 4a. A single capillary slot with a width of $1.4 \mathrm{~mm}$ is formed between a polished copper wall kept at a constant temperature and a heater wall, which consists of a $4 \mathrm{~mm}$ thick infrared-transparent $\mathrm{CaF}_{2}$ glass substrate with a three-layer coating. The first coating layer is a CrN black layer, enhancing the surface emissivity, and the second layer is a pure chromium layer acting as a resistance heater. Each of these two layers has a thickness of approximately $400 \mathrm{~nm}$. The third layer consists of nanofibers formed by electrospinning of $9 \mathrm{wt} \%$ Polyacrylonitrile solution (PAN; Mw = $150 \mathrm{kDa}$ ) in N,N-dimethylformamide (DMF). The nanofibers were electrospun for $60 \mathrm{~s}$ onto the chromium-coated substrate (see Figure $4 \mathrm{~b}$ ). The nanofiber mat thickness was 50-70 $\mu \mathrm{m}$, and the fiber diameter varied in the range 200-300 $\mathrm{nm}$. The SEM image of the nanofiber mat deposited over the glass substrate is depicted in Figure 4c. To improve the adhesion of the nanofiber mat to the glass substrate and prevent its delamination, the edges of the nanofiber mat were wetted with ethanol at the sidewalls. 
The temporal evolution of temperature at the backside of the black layer was recorded by an infrared (IR) camera with a frame rate of $1000 \mathrm{~Hz}$, spatial resolution of $29.27 \mu \mathrm{m} /$ pixel and a field of view of 224 pixel x 224 pixel. The IR camera was calibrated in situ. The two-dimensional temperature distribution was then used for computation of the time-dependent distribution of the heat flux transferred from the heater surface to the fluid (liquid or vapour). The calibration process and the method for determination of the local heat flux are described in Fischer et al. (2015). The quantitative difference in the heat flux distribution for a non-moving meniscus is quantified in Figure 5, in which the heat flux line profiles for non-moving menisci for the plain chromium surface and chromium surface with nanofibers are shown as functions of the vertical coordinate. In this diagram, the coordinate origin has been placed at the position of the maximal heat flux. The data have been spatially averaged over 184 pixel rows and time-averaged over 250 frames. The maximal heat flux on the nanofiber-coated surface exceeds the maximal heat flux on the plain chromium surface by approximately $60 \%$. In addition, the region of the higher heat flux is about $8 \%$ wider in the case of the coated surface than for plain chromium surfaces. Both results indicate a significant heat transfer enhancement due to the nano-textured heater surface.

(a)

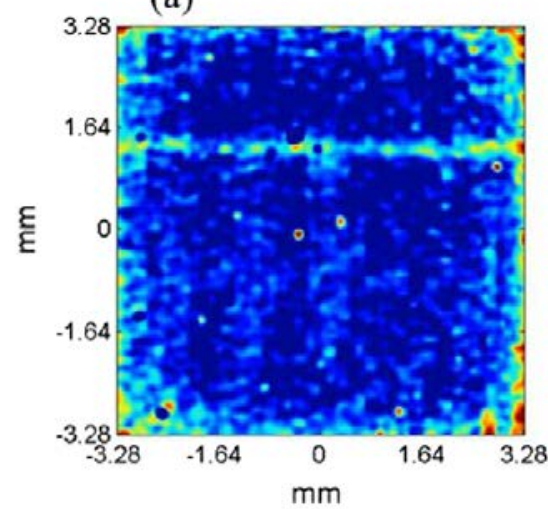

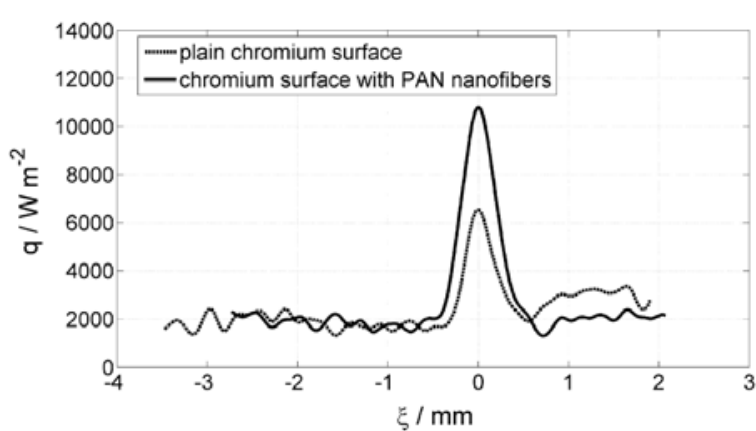

Figure 5: Heat flux profiles for a non-moving liquid-vapour interface at the saturation temperature $T_{s}=53.6 \pm 0.6^{\circ} \mathrm{C}$ and the average wall superheat $\Delta T_{w}=4.4 \pm 0.2 \mathrm{~K}$ (b)

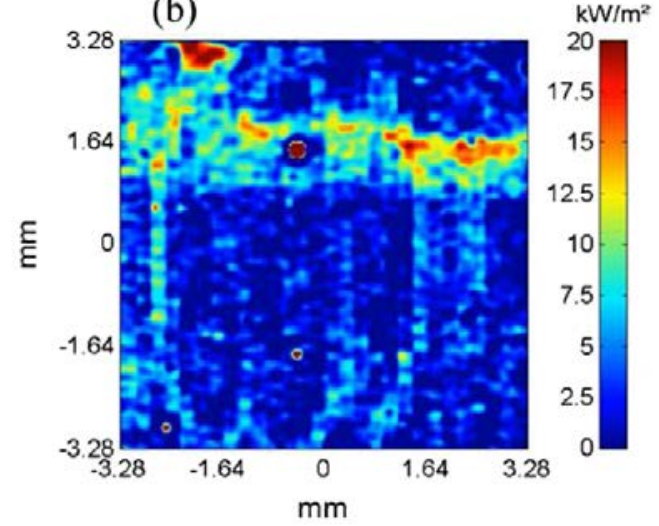

Figure 6: Heat flux fields for a receding meniscus. (a) Plain chromium surface; (b) chromium surface coated with PAN nanofiber mat. The meniscus receding velocity in both cases is $10 \mathrm{~mm} / \mathrm{s}$. The saturation temperature is $T_{s}=$ $52.9 \pm 0.6^{\circ} \mathrm{C}$ and the average wall superheat $\Delta T_{w}=3.6 \pm 0.2 \mathrm{~K}$.

The two-dimensional heat flux distribution at the solid-fluid interface for the receding meniscus is shown in Figure 6. If the substrate is not coated with nanofibers, the region of high heat flux is sharply localized around the apparent threephase contact line, showing qualitatively the same behaviour as for the non-moving meniscus. In contrast, if the substrate is coated, the region of high heat flux is distributed over an extended area. This effect is illustrated in Figure 7, showing the heat flux profiles at different time instants. In this figure, the data for each time instant have been averaged over 184 pixel rows. It is clearly seen that the region of high heat flux having a length of about 1-1.5 mm moves with approximately constant velocity in the direction of

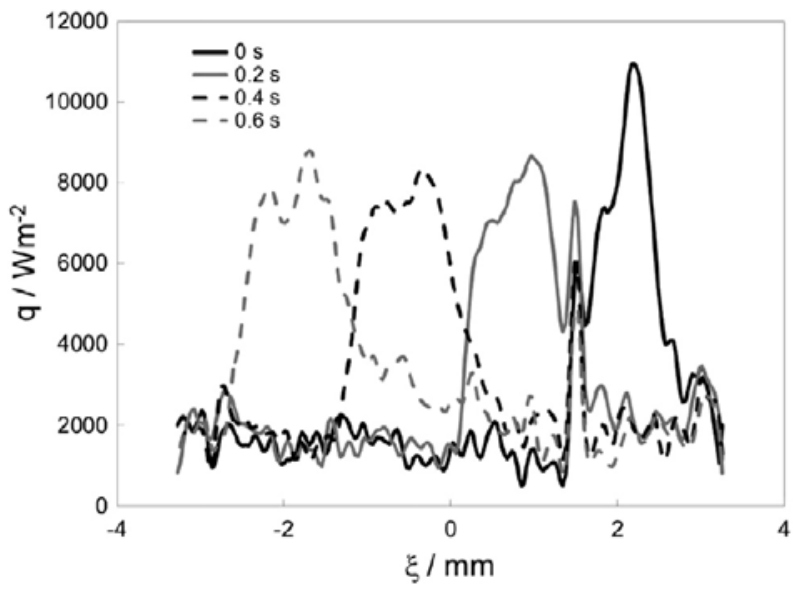

Figure 7: Heat flux profiles at different time instances for a receding meniscus on a chromium surface coated with PAN nanofiber mat. The saturation temperature $T_{s}=52.9 \pm 0.6^{\circ} \mathrm{C}$ and the average wall superheat $\Delta T_{w}=3.6 \pm 0.2 \mathrm{~K}$. The meniscus receding velocity is $10 \mathrm{~mm} / \mathrm{s}$. 
receding motion of the meniscus. It can be suggested that the receding meniscus leaves behind a region, in which the pores within the mat are fully or partially filled with the evaporating liquid. This effect is caused by strong capillary forces exerted by the porous structure and by the viscous forces decelerating the dewetting flow.

Evaporation of liquid at single non-moving, receding and advancing menisci on a nanofiber-coated heater surface has been investigated experimentally. The heat flux distribution has been determined from the temperature field measured by infrared thermography. We have shown that the evaporation near a non-moving meniscus in the presence of nanofibers results in the enhancement of maximal heat flux transferred from the heater by about $60 \%$ and in an increased length of high heat flux area by about $8 \%$. We have confirmed experimentally that the receding meniscus on a coated heater surface leaves behind a broad wetted region. The deposited liquid layer evaporates and thus significantly contributes to higher heat transfer rate. The behaviour of a single evaporating meniscus formed near a heater with nanofiber coating explains the mechanisms of heat transfer enhancement recently observed for two-phase flows on heated nanofiber-coated surfaces.

\section{Transient effects in ice nucleation of a water drop impacting onto a cold substrate [13] (TP-C3) Schremb, Roisman, Tropea}

The impact of supercooled water drops and subsequent icing of the impact surface is a severe hazard for transportation such as air traffic, shipping and road traffic, but is also a frequent problem for power supply systems and wind turbines. It is a highly complex process comprising several physical mechanisms from hydrodynamics and thermodynamics. Drop impact may be accompanied by heat transfer due to non-isothermal drop impact [14], is followed by nucleation of the liquid [13] and finally results in solidification of the liquid which is potentially influenced by the solid wall [15]. Due to its stochastic nature based on Brownian motion of molecules, nucleation is the most complex process involved, and is influenced by many different parameters such as the liquid temperature, the impact conditions, fluid flow and heat transfer during impact, the impact surface morphology, chemical properties of the impinging drop, etc.

So far, nucleation of supercooled water has only been investigated for simple configurations such as a liquid at rest, or in a defined shear flow or temperature gradient. Within the CRC, we've examined the stochastic nature of nucleation for the first time in the context of the complexity of a drop impact. Therefore, impact experiments have been performed numerous times under constant conditions to allow statistical analysis. It has been shown that in contrast to the nucleation rate of sessile drops, the nucleation rate during drop impact is not constant in time. It is the highest directly after impact and decreases with time. Based on a statistical nucleation model derived from Poisson statistics, the average number of nucleation sites per unit area on the wetted surface has been calculated. As shown in Fig. 8 for the case of inclined drop impact onto polished aluminum (8a) and normal impact onto sandblasted glass $(9 \mathrm{~b})$, the number of nucleation sites per unit area of the wetted surface $\left(\lambda_{s}\right)$ increases with time. However, the rate of increase of the nucleation rate decreases with time, although classical nucleation theory predicts constant nucleation rates for constant conditions.

a)

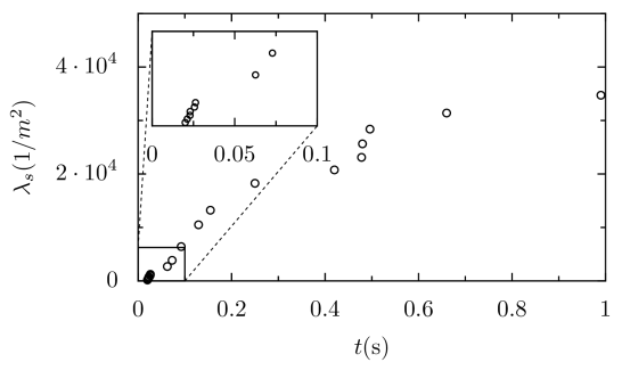

b)

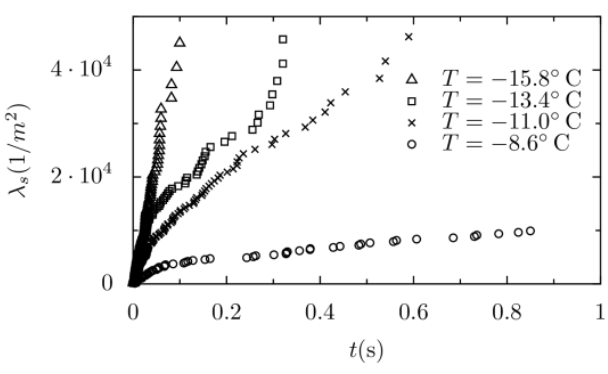

Figure 10. Evolution of the average number of nucleation sites per unit area, $\lambda_{s}$, during drop impact. a) Impact of water drops at $14.3^{\circ} \mathrm{C}$ onto polished aluminum at $-17.0^{\circ} \mathrm{C}$. The drop diameter, impact velocity and impact angle are $3.09 \mathrm{~mm}, 4.09 \mathrm{~m} / \mathrm{s}$ and $30^{\circ}$, respectively. b) Normal impact of supercooled water drops onto a sandblasted glass surface at the same temperature as the drops. The drop diameter and impact velocity are $3.2 \mathrm{~mm}$ and $2.2 \mathrm{~m} / \mathrm{s}$, respectively.

The reason for this transient behavior of nucleation has been found by a comparison of the time scales of the physical processes involved. While all other processes take place on a time scale much smaller than the time during which nucleation significantly changes, only the dissolving of air bubbles, which are commonly known to serve as nucleation sites, may be identified as relevant for a change of the nucleation rate. These air bubbles are entrapped during spreading over the surface and their size is of the order of the size of the surface roughness elements. To proof this hypothesis, experiments have been repeated with degassed water drops. In the case of an impact of degassed water drops, air bubbles entrapped during impact are dissolved much faster than in the case of nondegassed drops, as shown in Fig. 9 a) and b) for spread drops 40 ms after normal impact onto sandblasted glass. Therefore, less nucleation sites are available in the case of degassed water, resulting in a lower nucleation rate and 
a slower decrease of the relative number of liquid drops over time, which is the average probability of a drop to be liquid, as shown in Fig. 9 c).
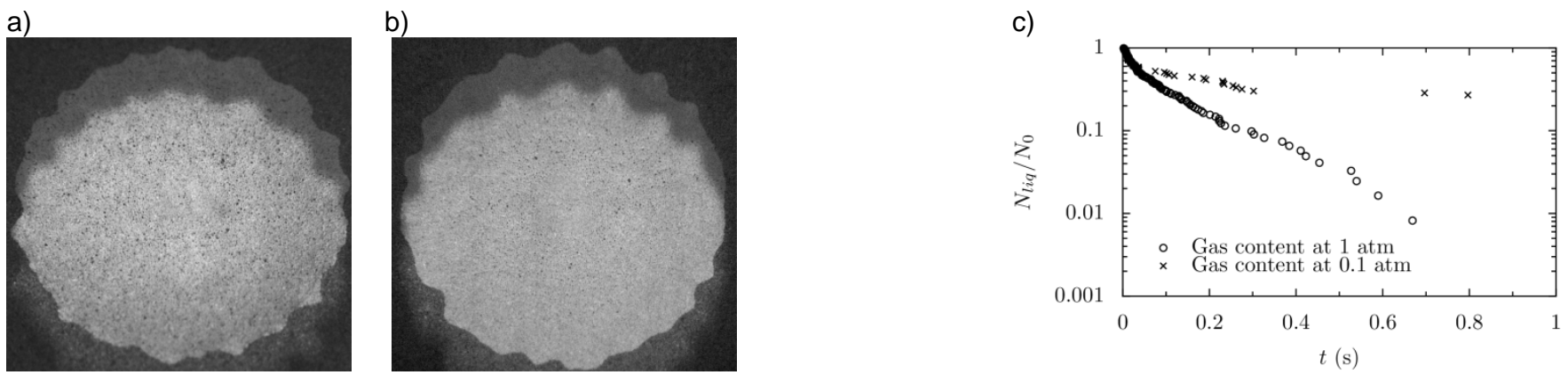

Figure 11. Influence of the liquid's gas content on nucleation of supercooled water drops after normal impact onto a sandblasted glass surface. The drop diameter is $3.2 \mathrm{~mm}$, the impact velocity is $2.2 \mathrm{~m} / \mathrm{s}$ and both the drops and the surface are at $-11^{\circ} \mathrm{C}$. a) Gas bubbles entrapped during drop spreading over the rough surface. Gas content according to saturation at $1 \mathrm{~atm}$. b) Less gas bubbles due to increased dissolving of bubbles in the case of degassed drops. Gas content according to saturation at $0.1 \mathrm{~atm}$. c) Temporal evolution of the relative number of liquid drops $N_{\text {liq }} / N_{0}$.

\section{Conclusions}

This paper has shown the structure of and the work carried out in the Collaborative Research Center SFB-TRR 75: "Droplet Dynamics under Extreme Ambient Conditions". The work in the current funding period of this CRC focuses on single drops and small groups of drops. Future work will consider large number of drops and in many instances the behaviour and application of sprays under extreme ambient conditions. Also further projects involving industrial participation are planned.

\section{Acknowledgements}

The members of the SFB-TRR 75 would like to thank the Deutsche Forschungsgemeinschaft (DFG) for the financial support of the SFB-TRR 75 .

\section{References}

[1] Plateau, J.A.F., Statique. Gauthier \& Villars, Paris (1873).

[2] Rayleigh, Lord J.S.W., Proc. London Math. Soc. 10, pp. 4-13 (1878).

[3] Pruppacher, H.R. and Klett, J.D., Microphysics of Clouds and Precipitation. Reidel Publ. , Dordrecht (1978).

[4] Lefebvre, A.H., Atomization and Sprays. Combustion: An International Series. Hemisphere Publishing Corporation, New York (1989).

[5] A. Frohn, N. Roth., Dynamics of Drops. Springer-Verlag, Heidelberg (2000).

[6] Weigand, B. Tropea, C., and Birkefeld, A., ILASS-Europe 2014, Bremen, Germany (2014)

[7] Tropea, C., and Weigand, B., ICLASS 2015, 13th Int. Conf. on Liquid Atomization and Spray Systems, Tainan, Taiwan (2015)

[8] Weigand, B., Tropea, C. and Schulte, K., ILASS-Europe, Brighton, UK (2016)

[9] Ouedraogo, Y., Gjonaj, E., Weiland, T., De Gersem, H., Steinhausen, C., Lamanna, G., Weigand, B., Preusche, A., Dreizler, A. and Schremb, M. Int. J. Heat and Fluid Flow 64:120-128 (2017).

[10] Weckenmann, E., Bork, B., Oldenhof, E., Lamanna, G., Weigand, B., Boehm, B., Dreizler, A. Zeitschrift für Phys. Chemie 225:1417-1431 (2011).

[11] Fischer, S., Sahu, R.P., Sinha-Ray, S., Yarin, A.L., Gambaryan-Roisman, T. and Stephan, P. Int. J. Heat and Mass Transfer 108:2444-2450 (2017).

[12] Fischer, S., Gambaryan-Roisman, T. and Stephan, P. Int. J. Heat Mass Transfer 88: 346-356 (2015)

[13] Schremb, M., Roisman, I. V. and Tropea, C. Phys Rev E 95: 022805 (2017).

[14] Schremb, M., Borchert, S., Berberovic, E., Jakirlic, S., Roisman, I.V. and Tropea, C. Int. J. Heat and Mass Transfer to appear (2017).

[15] Schremb, M. and Tropea, C. Phys Rev E 94: 052804 (2016). 